\title{
Tissue Responses to Stent Grafts with Endo-Exo-Skeleton for Saccular Abdominal Aortic Aneurysms in a Canine Model
}

\author{
Young Il Kim, $M D^{1}$, Young Ho Choi, $M D^{2}$, Jin Wook Chung, $M D^{1}$, Hyo-Cheol Kim, MD', Young Ho So, MD², \\ Hyun Beom Kim, $M D^{3}$, Seung-Kee Min, $M D^{4}$, Jae Hyung Park, $M D^{5}$ \\ ${ }^{1}$ Department of Radiology and Institute of Radiation Medicine, Seoul National University College of Medicine, Clinical Research Institute, Seoul \\ 110-744, Korea; ${ }^{2}$ Department of Radiology, Seoul National University Boramae Hospital, Seoul 156-707, Korea; ${ }^{3}$ Department of Radiology, National \\ Cancer Center, Goyang 410-769, Korea; ${ }^{4}$ Department of Surgery, Seoul National University College of Medicine, Seoul 110-744, Korea; ${ }^{5}$ Department \\ of Radiology, Gachon University Gil Medical Center, Incheon 405-760, Korea
}

Objective: We evaluated the effect of close contact between the stent and the graft on the induction of endothelial covering on the stent graft placed over an aneurysm.

Materials and Methods: Saccular abdominal aortic aneurysms were made with Dacron patch in eight dogs. The stent graft consisted of an inner stent, a expanded polytetrafluoroethylene graft, and an outer stent. After sacrificing the animals, the aortas with an embedded stent graft were excised. The aortas were inspected grossly and evaluated microscopically.

Results: The animals were sacrificed at two $(n=3)$, six $(n=3)$, and eight months $(n=2)$ after endovascular repair. In two dogs, the aortic lumen was occluded at two months after the placement. On gross inspection of specimens from the other six dogs with a patent aortic lumen, stent grafts placed over the normal aortic wall were covered by glossy white neointima, whereas, stent grafts placed over the aneurysmal aortic wall were covered by brownish neointima. On microscopic inspection, stent grafts placed over the normal aortic wall were covered by thin neointima $(0.27 \pm 0.05 \mathrm{~mm}$, mean \pm standard deviation) with an endothelial layer, and stent grafts placed over the aneurysmal aortic wall were covered by thick neointima $(0.62 \pm 0.17 \mathrm{~mm})$ without any endothelial lining. Transgraft cell migration at the normal aortic wall was more active than that at the aneurysmal aortic wall.

Conclusion: Close contact between the stent and the graft, which was achieved with stent grafts with endo-exo-skeleton, could not enhance endothelial covering on the stent graft placed over the aneurysms.

Index terms: Aorta; Aortic aneurysm; Stent-graft; Neointima; Endothelium

Received April 7, 2014; accepted after revision July 6, 2014. This study was supported by grant no. 04-2008-0180 from the Seoul National University Hospital Research Fund.

Corresponding author: Young Ho Choi, MD, Department of Radiology, Seoul National University Boramae Hospital, 20 Boramae-ro 5-gil, Dongjak-gu, Seoul 156-707, Korea.

- Tel: (822) 870-2534 - Fax: (822) 870-3863

- E-mail: cyho50168@naver.com

This is an Open Access article distributed under the terms of the Creative Commons Attribution Non-Commercial License (http://creativecommons.org/licenses/by-nc/3.0) which permits unrestricted non-commercial use, distribution, and reproduction in any medium, provided the original work is properly cited.

\section{INTRODUCTION}

After Parodi et al. (1) reported transfemoral intraluminal graft implantation for abdominal aortic aneurysms (AAAs), endovascular repair using stent grafts for aortic aneurysms has become a substitute for surgical vascular grafting and it is being widely used nowadays. In open surgery for aortic aneurysms, endothelialization of the luminal surface of vascular grafts occurs rarely $(2,3)$. Endovascular repair with stent grafts for aortic aneurysms does not cause any significant difference in endothelialization of the luminal 
surface (4).

A few studies assessing the tissue reaction to stent grafts have been performed in normal vessels or fusiform aneurysm models (4-11). When stent grafts were placed in the normal artery or aorta, follow-up examinations showed neointima with good endothelial covering that could be due to active transgraft cell migration. In contrast, neointima with poor endothelial covering was found when stent grafts were placed in fusiform aneurysm models (11).

Kim et al. (12) demonstrated the difference in neointimal covering after endovascular stent grafting between over normal aortic wall and over aneurysmal aortic wall in the same object using saccular aneurysm models. The models had both aneurysmal and normal aortic walls in the same cross section and the authors inserted endoskeleton stent grafts. In their study, they suggested that the development of neointima with poor endothelial covering over the aneurysmal aortic wall might be due to loose contact between the stent and the graft, which causes continuous free floating movement of the graft according to the pulsatile aortic flow. Minimizing the floating movement of the graft by achieving close contact between the stent and the graft might increase the chance of endothelialization of the luminal surface and stabilization free from thrombi.

Herein, we made stent grafts with endo-exo-skeleton to achieve close contact between the stent and the graft and evaluated their effect on the induction of formation of neointima with good endothelial covering on the stent graft placed over the aneurysms. We expected that the stent graft with endo-exo-skeleton could achieve close contact between the stent and the graft and enhance endothelial covering on the luminal surface of stent grafts placed over saccular aneurysms.

\section{MATERIALS AND METHODS}

\section{Aneurysm Models}

Under a protocol approved by the Clinical Research Institute, 8 adult mongrel dogs of 20 to $25 \mathrm{~kg}$ body weight were used for this experiment. Each animal was anesthetized with an intramuscular injection of $10 \mathrm{mg} /$ $\mathrm{kg}$ ketamine hydrochloride and xylazine hydrochloride. The animals were mechanically ventilated with enflurane gas after endotracheal intubation. Aneurysms were made with longitudinal incision of the abdominal aorta and attachment of Dacron patch after laparotomy. A saccular aneurysm-like patch was premade on the back table with
5-0 prolene running sutures. After applying vascular clamp on the infrarenal aorta, a 2-cm arteriotomy was made and the patch was anastomosed with 5-0 prolene sutures. Aortic clamping time was usually less than 10 minutes. The size of Dacron patch for the aneurysms was $2.0 \times 2.0 \mathrm{~cm}$ to $2.6 \times 2.6 \mathrm{~cm}$. After one week, CT angiography with threedimensional reconstruction was performed to confirm the aneurysm formation without complications, and to measure the aneurysm size. Mean length, depth, and width of the aneurysms were $16 \pm 2 \mathrm{~mm}, 12 \pm 1 \mathrm{~mm}$, and $12 \pm 1 \mathrm{~mm}$, respectively.

\section{Stent Grafts}

The stent graft (Fig. 1) consisted of implantable Nitinol stent (Comvi Stent; Taewoong Medical, Gimpo, Korea) and expanded polytetrafluoroethylene (ePTFE). The Nitinol wire was woven of closed wire to avoid sharp edges. The ComVi Stent has a unique sandwich structure. It has a double straight and round cylindrical inner stent and outer stent. The ePTFE graft was placed between the inner stent and the outer stent while exempting both ends. The Nitinol wire and ePTFE graft were bound together with Prolene 6-0 sutures (Ethicon ${ }^{\mathrm{R}}$, Ethicon Inc., Somervill, NJ, USA) and it had eight radiopaque markers at both ends to facilitate imaging through $\mathrm{X}$-ray photography.

The size of stent grafts was determined on the basis of CT angiography performed one week after surgical aneurysm formation. In all animals, the aortic diameters were $10 \pm 0.5 \mathrm{~mm}$ with identical values at both landing sites $10 \mathrm{~mm}$ proximal and distal to aneurysms. As the stent graft diameter is usually oversized by $20 \%$ over the proximal and distal landing zones, the stent graft diameter was determined to be $12 \mathrm{~mm}$ in all of the animals. Approximately 20-mm landing zones of the normal aorta were planned proximal and distal to the aneurysms. Considering that the length of the aneurysms was $16 \pm 2.0$ $\mathrm{mm}$ with little variation, a stent graft length of $60 \mathrm{~mm}$ was determined to be sufficient to cover the aneurysm in all of the animals.

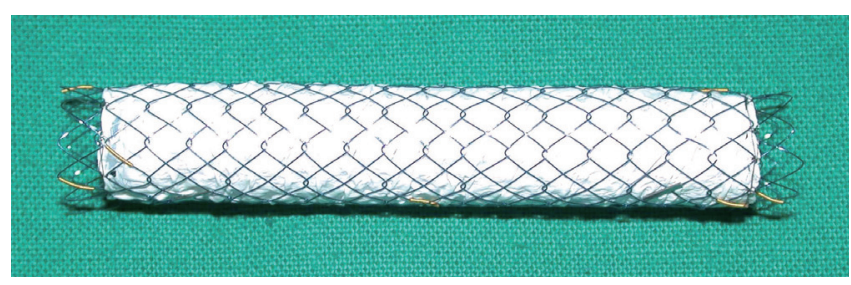

Fig. 1. Stent graft with endo-exo-skeleton consisting of inner bare stent, middle graft, and outer bare stent. 


\section{Animal Study}

Stent grafts were inserted one month after aneurysm formation. The right common carotid artery was incised, and a 10-Fr sheath was inserted. Heparin (100 units/ $\mathrm{kg}$ ) was injected intravenously to prevent thrombosis during the procedure. Stent grafts were deployed in the infrarenal aorta with saccular aneurysms after angiography. Completion angiography was performed to demonstrate complete exclusion of the aneurysm without any endoleak.

CT angiography was performed to observe luminal patency of the aorta just before the procedure, at two, six, and eight months after endovascular stent grafting. When the luminal diameter at the mid-portion of the aneurysm was no less than $100 \%$ of the luminal diameter at the level 10 $\mathrm{mm}$ proximal and distal to the aneurysm, the stent graft was considered to be patent.

\section{Histopathologic Evaluation}

The animals were euthanized with an overdose of thiopental sodium. Ten thousand units of heparin were administered intravenously just before death to prevent thrombosis while harvesting the aortic specimen. The

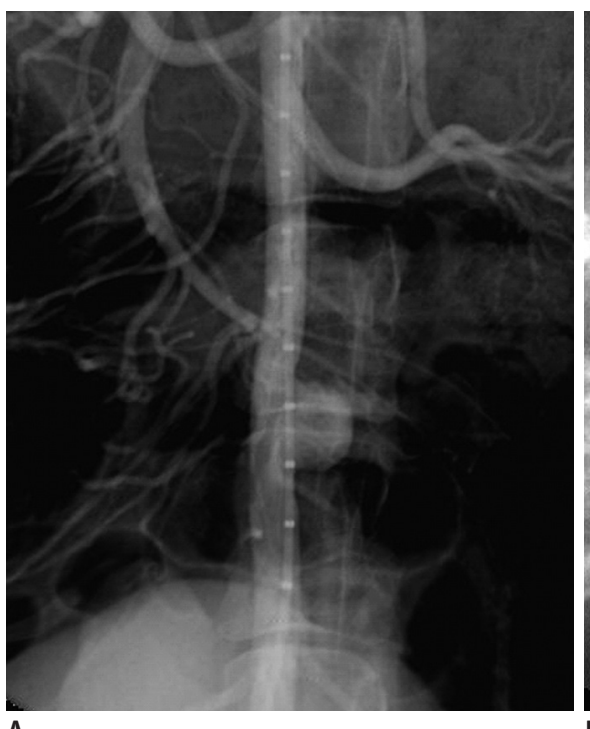

\section{A}

Fig. 2. Angiography before and after stent graft placement.
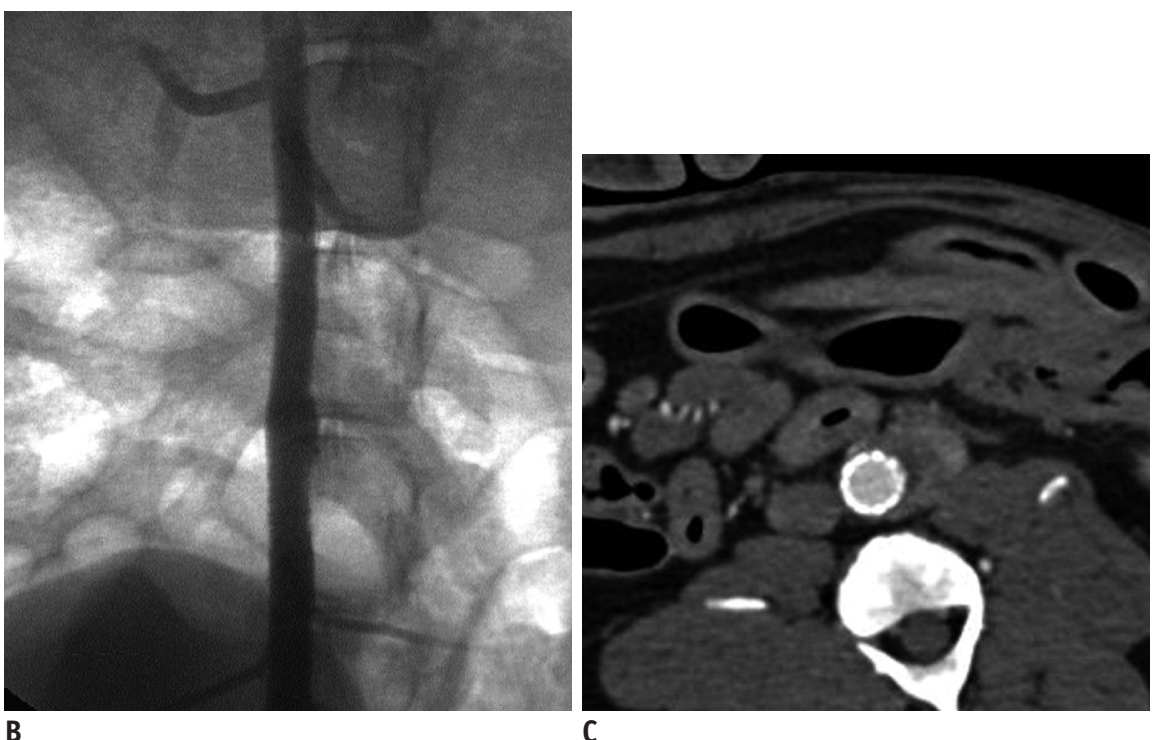

A. Conventional angiography shows aortic saccular aneurysm to left of aorta. B. On completion angiography after stent graft placement, middle part of inserted stent graft shows outward convexity towards saccular aneurysm. C. CT angiography at two months demonstrates complete exclusion of aneurysm, no endoleak, and patent lumen.

Table 1. Summary of Results after Endovascular Stent Grafting in Eight Dogs with Saccular Aneurysm

\begin{tabular}{|c|c|c|c|c|c|c|c|c|}
\hline \multirow{2}{*}{ Number } & \multirow{2}{*}{$\begin{array}{l}\text { Indwelling } \\
\text { Time }\end{array}$} & \multirow{2}{*}{ Patency } & \multicolumn{2}{|c|}{ Gross Inspection of Neointima } & \multicolumn{2}{|c|}{ Transgraft Cell Migration } & \multicolumn{2}{|c|}{$\begin{array}{c}\text { Endothelial Covering } \\
\text { on Neointima }\end{array}$} \\
\hline & & & Over Aneurysm & $\begin{array}{l}\text { Over Normal } \\
\text { Aorta }\end{array}$ & $\begin{array}{c}\text { Over } \\
\text { Aneurysm }\end{array}$ & $\begin{array}{l}\text { Over Normal } \\
\text { Aorta }\end{array}$ & $\begin{array}{c}\text { Over } \\
\text { Aneurysm }\end{array}$ & $\begin{array}{c}\text { Over Normal } \\
\text { Aorta }\end{array}$ \\
\hline 1 & 2 months & Occluded & & & Grade 1 & Grade 1 & & \\
\hline 2 & 2 months & Occluded & & & Grade 1 & Grade 1 to 2 & & \\
\hline 3 & 2 months & Patent & Brown, not covering stent wire & Glossy, white & Grade 1 & Grade 2 & - & + \\
\hline 4 & 6 months & Patent & Brown, partially covering stent wire & Glossy, white & Grade 2 & Grade 3 to 4 & - & + \\
\hline 5 & 6 months & Patent & Brown, partially covering stent wire & Glossy, white & Grade 2 & Grade 3 & - & + \\
\hline 6 & 6 months & Patent & Brown, not covering stent wire & Glossy, white & Grade 2 & Grade 3 & - & + \\
\hline 7 & 8 months & Patent & $\begin{array}{l}\text { Thick, brown, protruding toward } \\
\text { aortic lumen }\end{array}$ & Glossy, white & Grade 3 & Grade 4 & - & + \\
\hline 8 & 8 months & Patent & $\begin{array}{l}\text { Thick, brown, protruding toward } \\
\text { aortic lumen }\end{array}$ & Glossy, white & Grade 1 & Grade 3 & - & + \\
\hline
\end{tabular}

Note. - Grade 1 = no collagen and no spindle cell, Grade 2 = collagen and less than 10 spindle cells, Grade $3=$ collagen and 10 to 50 spindle cells, Grade $4=$ collagen and more than 50 spindle cells 
abdominal aorta with a stent graft was removed en bloc, flushed with heparinized saline, and fixed with $10 \%$ formalin solution. The fixed aorta was cut longitudinally along the opposite side of the aneurysm for visual inspection. Luminal patency and neointimal covering were examined.

After gross inspection, the aorta was bisected with another longitudinal cut of the aorta. After manual removal of stent wires from the harvested aortas, a transected slice of $5 \mathrm{~mm}$ thickness perpendicular to the long axis of the stent graft that included the aneurysm was obtained, mounted on glass slides after manual removal of the stent wire, and examined with hematoxylin-eosin staining, Masson's trichrome staining, elastic fiber staining, smooth muscle actin immunohistochemical staining, and Factor VIII-related antigen immunohistochemical staining under light microscopy.

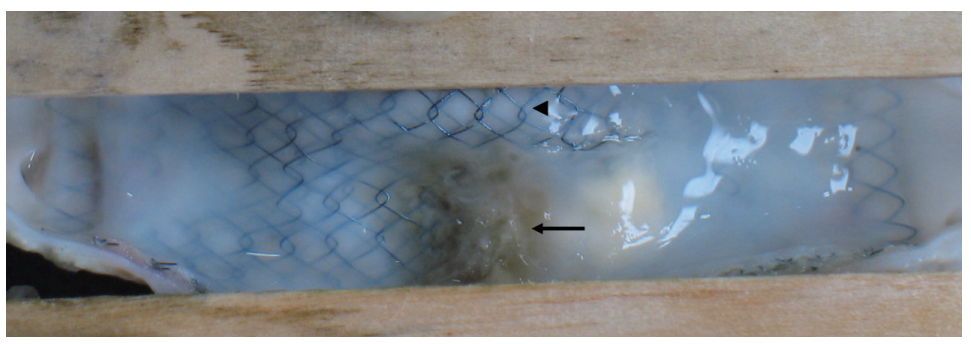

A

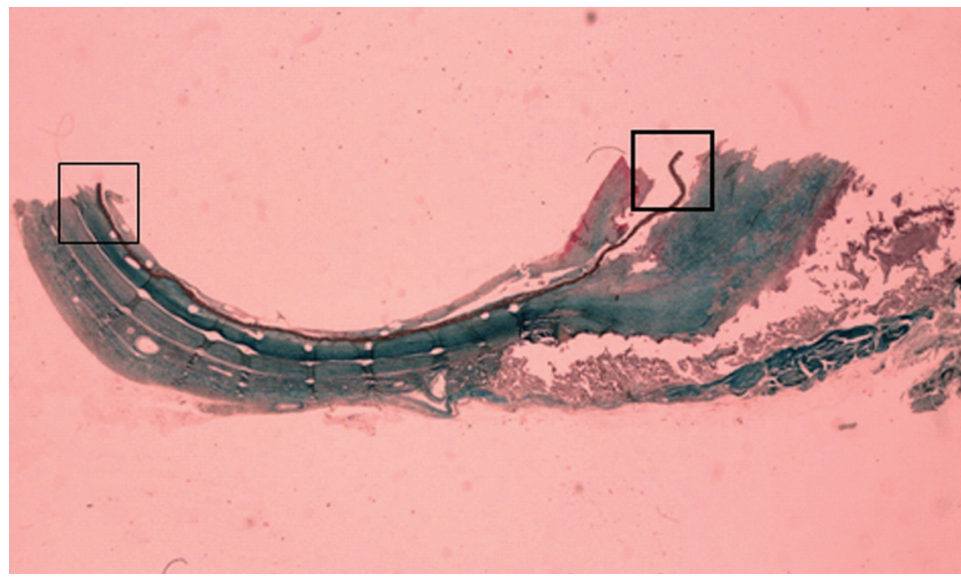

B

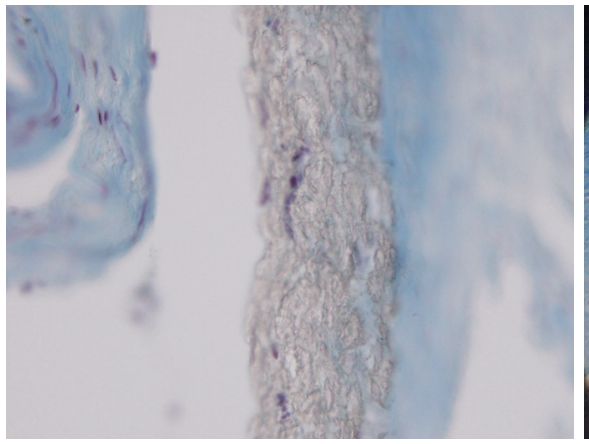

D
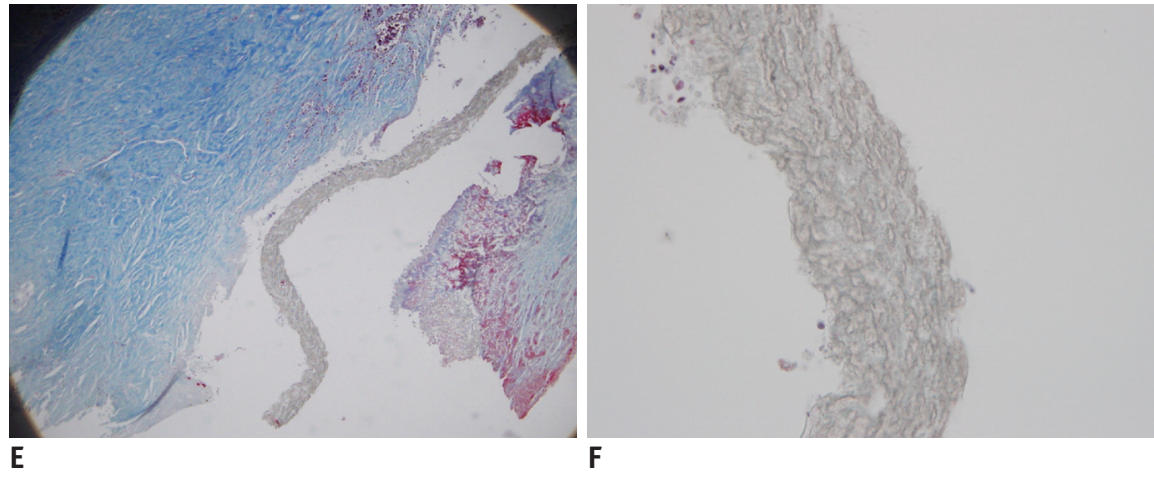

$\mathbf{F}$

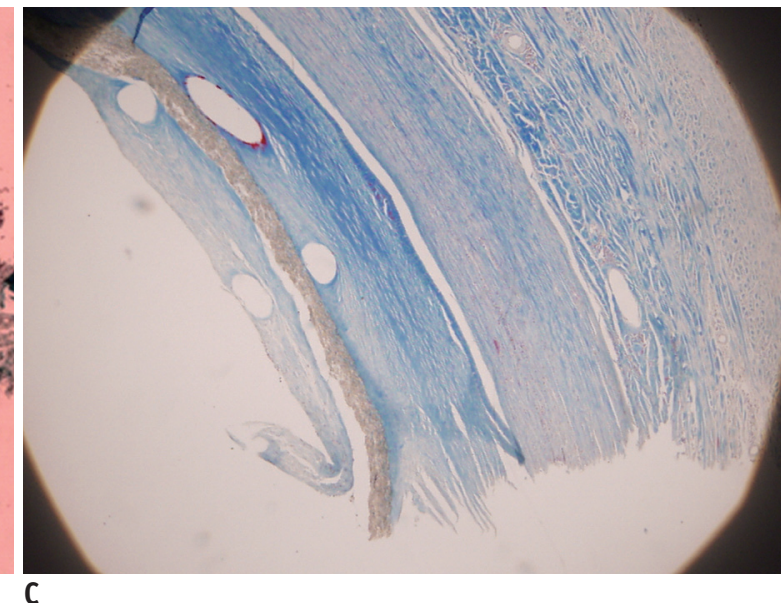

C

Fig. 3. Gross inspection findings and transgraft cell migration.

A. Gross inspection after longitudinal incision of abdominal aorta at opposite side of aneurysm demonstrates thick brown neointima (arrow) over aneurysmal aortic wall, which protrudes towards aortic lumen. Glossy white neointima (arrowhead) is noted over normal aortic wall. B. Microscopic examination (Masson's trichrome stain, original magnification $\times 1$ ) shows thin neointima over normal aortic wall (thin-lined rectangular block) and thick neointima over aneurysmal aortic wall (thick-lined rectangular block). C, D. Magnified view of thin-lined rectangular block in B located in normal aortic wall (original magnification, $x 40$ and $\times 400$ ) shows graft with lot of collagen (bright blue) and more than ten spindle cells (violet) rated as Grade 3. Brown band between footprints of inner and outer stent wire struts in C and E is graft. E, F. Magnified view of thick-lined rectangular block in B located in stent graft placed over excluded saccular aneurysm (original magnification, $\mathrm{x} 40$ and $\mathrm{x} 400$ ) shows graft with little collagen and few spindle cells rated as Grade 1. 
During microscopic inspection, we examined the distance between the stent and the graft, thickness of the neointima, transgraft cell migration, endothelial covering on the neointima formed over the aneurysmal and normal aortic walls. The distance measured was the maximal distance between the center of the inner stent wire and the inner surface of the graft. The thickness of neointima was measured at the thickest portion over the aneurysmal and normal aortic walls. Transgraft cell migration was graded as follows: grade 1, no collagen and no spindle cells on 400 times magnification of Masson's trichrome staining; grade 2, collagen and less than 10 spindle cells; grade 3, collagen and 10 to 50 spindle cells; and grade 4, collagen and over fifty spindle cells (12). Spindle cells with brownish cytoplasm on smooth muscle actin immunohistochemical staining were considered as cells with myoid differentiation. We assessed whether the neointima was covered with an endothelial layer on 200 to 400 times magnification. Lining cells with brownish cytoplasm on Factor VIII-related antigen immunohistochemical staining were considered as endothelial cells because the antigen has been firmly established as an endothelial cell marker in canines.

\section{RESULTS}

Angiography (Fig. 2) performed immediately after stent

Table 2. Comparison of Distance between Stent and Graft and Neointimal Thickness between Two Studies Using Different Types of Endovascular Stent Grafts

\begin{tabular}{lccccccc}
\hline & \multicolumn{2}{c}{ Distance between Stent and Graft (mm) } & & \multicolumn{2}{c}{ Neointima Thickness (mm) } \\
\cline { 2 - 3 } \cline { 5 - 6 } & Over Saccular Aneurysm & Over Normal Aortic Wall & Over Saccular Aneurysm Over Normal Aortic Wall \\
\hline Kim et al. (12) (exoskeleton type) & $1.15 \pm 0.64$ & $0.10 \pm 0.02$ & & $1.80 \pm 0.89$ & $0.60 \pm 0.59$ \\
Present study (endo-exo-skeleton type) & $0.19 \pm 0.05$ & $0.12 \pm 0.03$ & & $0.62 \pm 0.17$ & $0.27 \pm 0.05$ \\
\hline
\end{tabular}
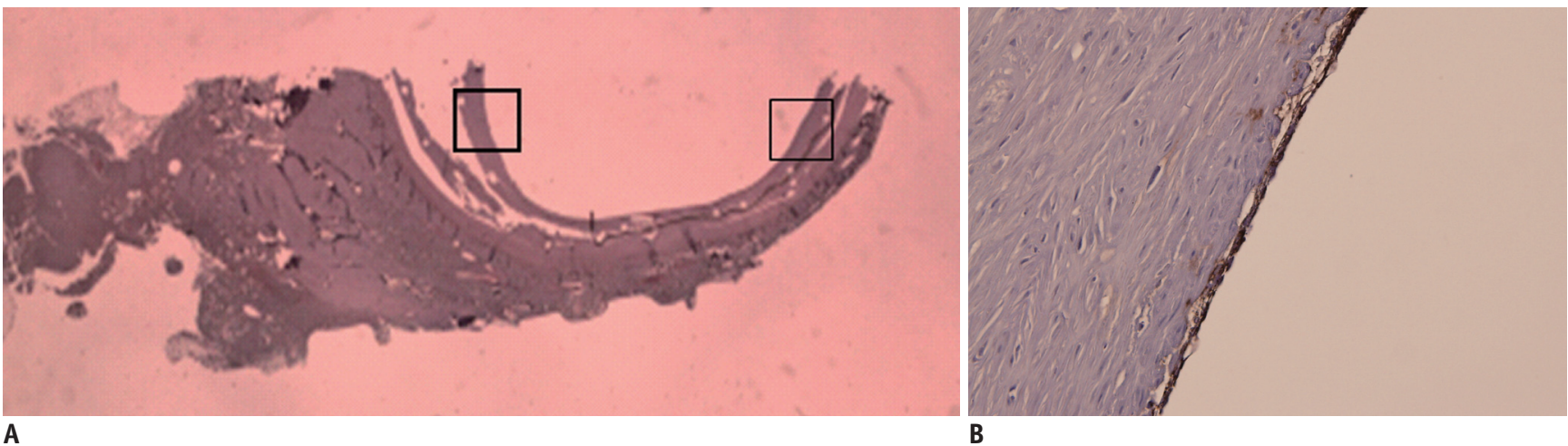

A

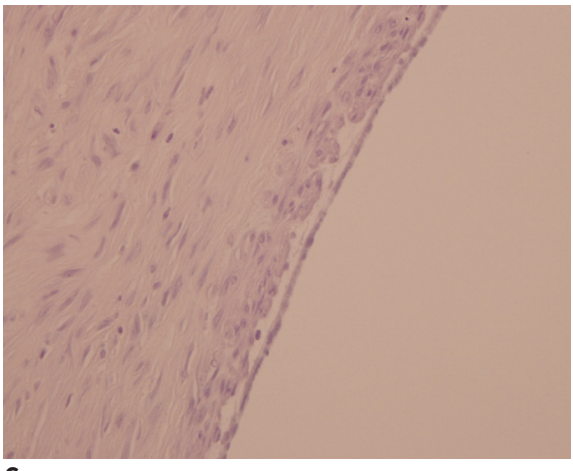

C

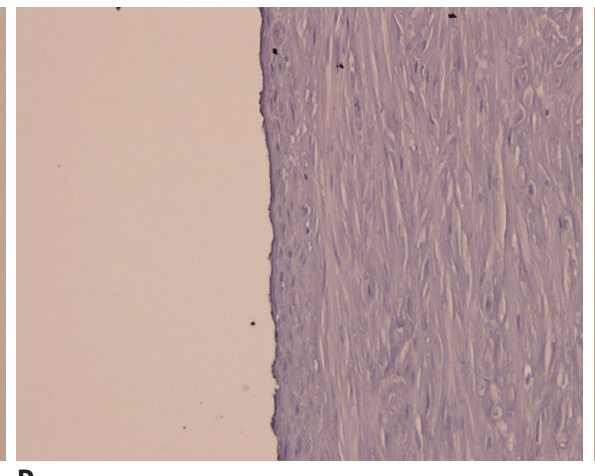

D

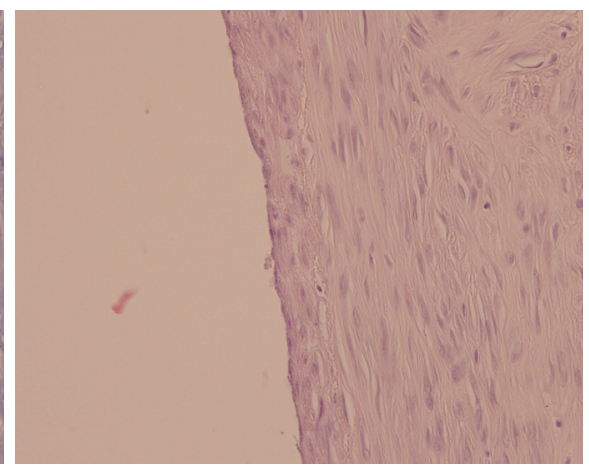

E

Fig. 4. Immunohistochemical staining of endothelial covering.

A. Cross-section of bisected aorta contains stent graft and aneurysm (Factor VIII-related antigen immunohistochemical staining, original magnification $\times 1$ ). Thin-lined rectangular block is normal aortic wall and thick-lined rectangular block is aneurysmal aortic wall. B, C. Magnified view of thin-lined rectangular block in A located in normal aorta (B; Factor VIII-related antigen immunohistochemical staining, C; HematoxylinEosin staining, original magnification, $\times 400$ ) shows layer of cells strongly reactive to Factor VIII-related antigen immunohistochemical staining, which indicates endothelial covering on neointima. D, E. Magnified views of thick-lined rectangular block in A located in stent graft placed over excluded saccular aneurysm (D; Factor VIII-related antigen immunohistochemical staining, E; Hematoxylin-Eosin staining, original magnification, $x$ 400) show layer of cells in neointima that are not stained on Factor VIII-related antigen immunohistochemical staining. 
graft placement demonstrated the accurate location of stent graft and complete exclusion of the aneurysm in all the eight dogs, and stent grafts placed over the aneurysmal aortic wall protruded outwards and resembled a barrel. CT angiography at two months demonstrated aortic occlusion in two dogs and they were sacrificed (Table 1). In the other six dogs, CT angiography showed a patent lumen and complete exclusion of the aneurysm. Afterwards, there was no further aortic occlusion.

All of the dogs were sacrificed to obtain aortic specimens at two $(n=3)$, six $(n=3)$, and eight $(n=2)$ months after endovascular stent grafting, respectively. $0 \mathrm{n}$ gross inspection, the findings of aortic occlusion in two dogs and patent lumen in the other six dogs observed on CT angiography were confirmed. Luminal surface of stent grafts placed over the normal aortic wall was covered by glossy white neointima through which the stent was visualized (Fig. 3). In contrast, luminal surface of stent grafts placed over the aneurysmal aortic wall was covered by brownish neointima that did not cover the stent wire $(n=2)$, brown neointima that partially covered the stent wire $(n=2)$, and thick brownish neointima that protruded towards the aortic lumen $(n=2)$.

On microscopic inspection, the distance between the stent and the graft in dogs was $0.19 \pm 0.05$ (mean \pm standard deviation) $\mathrm{mm}$ and $0.12 \pm 0.03 \mathrm{~mm}$ at the aneurysmal aortic wall and the normal aortic wall, respectively (Table 2). Neointimal thickness in dogs was $0.62 \pm 0.17 \mathrm{~mm}$ and 0.27 $\pm 0.05 \mathrm{~mm}$ at the aneurysmal aortic wall and the normal aortic wall, respectively. Distance between the stent and the graft and neointimal thickness at the aneurysmal aortic wall tended to be greater than those at the normal aortic wall.

Transgraft cell migration (Fig. 3) at the normal aortic wall was active, and the migration at the aneurysmal aortic wall was less active. 0 n smooth muscle actin immunohistochemical staining, some spindle cells in the graft were stained brown and the other cells were not stained. This indicates that cells with myoid differentiation got along with cells with fibroid differentiation in the graft.

The luminal surface of stent grafts placed over the normal aortic wall was covered with a endothelial layer (Fig. 4), and the luminal surface of stent grafts placed over the aneurysmal aortic wall was partially covered with a layer of cells that were not stained on Factor VIII-related antigen immunohistochemical staining.

There was no difference in the endothelial covering between the two-, six-, and eight-month follow-up groups; complete endothelial covering on the normal aortic wall and no endothelial covering on the saccular aneurysm.

\section{DISCUSSION}

Endothelial covering on the neointima is crucial for the stabilization of neointima after endovascular grafting (2, $4,13,14)$. Poor endothelial covering induces adherence of thrombi to the luminal surface of stent grafts and thick cellular proliferation. Endoskeleton stent grafts used in the study by Kim et al. (12) did not have endothelial covering on the brownish thick neointima on the stent grafts placed over the aneurysmal aortic wall. The thrombi on the luminal surface of stent grafts were still fresh even at six months after implantation, which implies that there was continuous recirculation between blood flow and thrombi adherent to the luminal surface of stent grafts. With respect to the cause of delayed endothelialization, it was postulated that disjoint stent and graft could prevent the formation of endothelial covering due to continuous movement of the graft, disturbance in the aortic blood flow, and difficulty in transgraft cell migration.

In this study, the use of stent grafts with endo-exoskeleton induced close contact between the stent and the graft over the aneurysmal aortic wall. In the study by Kim et al. (12) using endoskeleton stent grafts, the distance between the stent and the graft in dogs was $1.15 \pm 0.64$ $\mathrm{mm}$ and $0.10 \pm 0.02 \mathrm{~mm}$ at the aneurysmal aortic wall and normal aortic wall, respectively. On comparing this result with our result, it was observed that there was a remarkable decrease in the distance between the stent and the graft at the aneurysmal aortic wall in our study (Table 2). Despite achieving close contact between the stent and the graft at the aneurysmal aortic wall by the stent grafts used in this study, we failed to demonstrate endothelial covering of stent grafts placed over the aneurysmal aortic wall in any of the dogs up to the 8-month follow-up. Instead, the luminal surface of the stent grafts placed over the aneurysmal aortic wall was covered with brownish thick neointima without any evidence of endothelial lining, which was similar to the finding in the study by Kim et al. (12). The neointima over the aneurysmal aortic wall can be called 'pseudointima' because it did not have a layer of mature endothelial cells that pathologically confirms the presence of true intima. The brownish discoloration is caused by the fresh and organized blood components in the pseudointima, which 
indicates that the pseudointima covering the stent grafts placed over the aneurysmal aortic wall remains immature and thrombogenic until 8 months after implantation.

Ombrellaro et al. (15) reported that endothelial covering on Palmaz balloon-expandable stent grafts was significantly greater than that on interposition grafts. Endovascular grafting provided enhanced endothelialization than open repair. Therefore, transgraft cell migration could be an important route for obtaining endothelial covering on the neointima. In this study, a lot of spindle cells and collagen in the graft placed over the normal aortic wall indicated active transgraft cell migration. Transgraft cell migration at the stent grafts placed over the aneurysmal aortic wall was less active than that at the stent grafts placed over the normal aortic wall, and this finding was consistently observed in both the previous (12) and current studies. Therefore, impaired transgraft cell migration might be a cause of poor or delayed endothelialization. Endothelialization may require or can be facilitated by an environment with healthy supporting structures with sufficient oxygen and nutrient supply. We think that high amount of collagen and spindle cells within the graft in the pathologic specimens represents the presence of a good environment for endothelialization, and in contrast, sparse amount of collagen and spindle cells represent the presence of an inadequate environment. According to the transgraft cell migration theory, the neointima is formed by migration and proliferation of cells from the media, and we can expect that cell migration through the graft placed over the aneurysmal aortic wall may be impaired as there was a gap filled with thrombi between the inserted stent grafts and the aneurysmal aortic wall. In addition, the saccular aneurysm was made with the synthetic Dacron graft.

Although the thrombi were eventually replaced by organized tissue, the environment in the organized aneurysmal sac might not be as good as that in the media of the healthy aorta.

Another route of obtaining endothelial covering might be cell migration along the luminal surface from the normal aorta to the grafted aorta. A conventional surgery for aneurysms using interpositional PTFE graft (16) showed that both graft ends of 1 to $1.5 \mathrm{~cm}$ anchored to the aorta were endothelialized, but the rest middle graft was not endothelialized. Endothelial covering over the aneurysmal aortic wall might also occur due to cell migration along the luminal surface from the adjacent part of stent grafts placed over the normal aortic wall with complete endothelial covering of the neointima due to transgraft cell migration. With this mechanism, it could take a longer time for the neointima over the aneurysmal aortic wall to be endothelialized. However, complete endothelial covering on the neointima over the normal aortic wall was achieved at two months, and there was no evident progress in the endothelial covering over the aneurysmal aortic wall until eight months. It could be inferred that cell migration along the luminal surface might not be significant for obtaining endothelial covering on the luminal surface of the stent grafts placed over the aneurysmal aortic wall.

Aneurysmal segments produce sluggish or turbulent blood flow, which can activate platelet activity and damage the endothelium $(17,18)$. In this study, the middle part of the inserted stent grafts showed an outward convexity towards the saccular aneurysm. This segment might produce turbulent flow similar to that in the aneurysmal aortic wall and prevent the formation of endothelial lining.

There were several limitations to our study. First, the sample size was small; therefore, a study with a larger number of animals is needed. Second, we did not obtain the long-term result after eight months. Accordingly, a study for achieving long-term results is needed. Third, we used the Dacron patch to make saccular aneurysms in this study. The patch was good in making aneurysms of the intended size and shape. However, it has some limitations as an aneurysm model because it does not expand naturally and does not contain aortic branches. This could cause a bias in evaluating the natural course of aneurysms and tissue reaction after endovascular stent grafting. There is a possibility that saccular aneurysms made with an autogenous vein patch may behave differently and show better endothelialization after endovascular stent grafting. Fourth, we used saccular AAAs instead of fusiform AAAs for evaluating the effect of close contact between the stent and the graft, and the results of this study using saccular AAAs might be different from those of studies using fusiform AAAs.

In conclusion, close contact between the stent and the graft in stent grafts with endo-exo-skeleton could not enhance endothelial covering on the stent graft placed over the aneurysms.

\section{REFERENCES}

1. Parodi JC, Palmaz JC, Barone HD. Transfemoral intraluminal graft implantation for abdominal aortic aneurysms. Ann Vasc 
Tissue Responses to Stent Grafts for Saccular AAAs in a Canine Model

Surg 1991;5:491-499

2. Noishiki Y, Tomizawa Y, Yamane Y, Matsumoto A. The vicious cycle of nonhealing neointima in fabric vascular prostheses. Artif Organs 1995;19:7-16

3. Berger K, Sauvage LR, Rao AM, Wood SJ. Healing of arterial prostheses in man: its incompleteness. Ann Surg 1972;175:118-127

4. Laborde JC, Parodi JC, Clem MF, Tio F0, Barone HD, Rivera $\mathrm{FJ}$, et al. Intraluminal bypass of abdominal aortic aneurysm: feasibility study. Radiology 1992;184:185-190

5. Dolmatch BL, Tio FO, Li XD, Dong YH. Patency and tissue response related to two types of polytetrafluoroethylenecovered stents in the dog. J Vasc Interv Radiol 1996;7:641649

6. Marty B, Leu AJ, Mucciolo A, von Segesser LK. Biologic fixation of polyester-versus polyurethane-covered stents in a porcine model. J Vasc Interv Radiol 2002;13:601-607

7. Wilson EP, White RA, Kopchok GE, Donayre CE, de Virgilio C, Geselschap JH, et al. Deployment and healing of an ePTFE encapsulated stent endograft in the canine aorta. Ann Vasc Surg 1997;11:354-358

8. Virmani R, Kolodgie FD, Dake MD, Silver JH, Jones RM, Jenkins $M$, et al. Histopathologic evaluation of an expanded polytetrafluoroethylene-nitinol stent endoprosthesis in canine iliofemoral arteries. J Vasc Interv Radiol 1999;10:445-456

9. Boudghène $F$, Anidjar $S$, Allaire $E$, Osborne-Pellegrin $M$, Bigot JM, Michel JB. Endovascular grafting in elastase-induced experimental aortic aneurysms in dogs: feasibility and preliminary results. J Vasc Interv Radiol 1993;4:497-504

10. Palmaz JC, Tio F0, Laborde JC, Clem M, Rivera FJ, Murphy KD, et al. Use of stents covered with polytetrafluoroethylene in experimental abdominal aortic aneurysm. J Vasc Interv Radiol 1995;6:879-885
11. Benson AE, Palmaz JC, Tio FO, Sprague EA, Encarnacion CE, Josephs SC. Polytetrafluoroethylene-encapsulated stentgrafts: use in experimental abdominal aortic aneurysm. J Vasc Interv Radiol 1999;10:605-612

12. Kim HB, Choi YH, So YH, Min SK, Kim HC, Kim YI, et al. Tissue responses to endovascular stent grafts for saccular abdominal aortic aneurysms in a canine model. $J$ Korean Med Sci 2012;27:1170-1176

13. Makutani S, Kichikawa K, Uchida H, Maeda M, Konishi $N$, Hiasa $Y$, et al. Effect of antithrombotic agents on the patency of PTFE-covered stents in the inferior vena cava: an experimental study. Cardiovasc Intervent Radiol 1999;22:232238

14. Ishii S, Sato M, Sonomura T, Yamada K, Tanihata H, Ishikawa $\mathrm{H}$, et al. Optimal covering material for stent-grafts placed in the portal vein in a canine model. Cardiovasc Intervent Radiol 2005;28:624-631

15. Ombrellaro MP, Stevens SL, Kerstetter K, Freeman MB, Goldman $\mathrm{MH}$. Healing characteristics of intraarterial stented grafts: effect of intraluminal position on prosthetic graft healing. Surgery 1996;120:60-70

16. Hanel KC, McCabe C, Abbott WM, Fallon J, Megerman J. Current PTFE grafts: a biomechanical, scanning electron, and light microscopic evaluation. Ann Surg 1982;195:456-463

17. Briguori C, Sarais C, Sivieri G, Takagi T, Di Mario C, Colombo A. Polytetrafluoroethylene-covered stent and coronary artery aneurysms. Catheter Cardiovasc Interv 2002;55:326-330

18. Yip HK, Chen MC, Wu CJ, Hang CL, Hsieh KY, Fang CY, et al. Clinical features and outcome of coronary artery aneurysm in patients with acute myocardial infarction undergoing a primary percutaneous coronary intervention. Cardiology 2002;98:132-140 